\title{
Genome-wide identification, characterization and expression analysis of populus leucine-rich repeat receptor-like protein kinase genes
}

Yanjun Zan, Yan Ji, Yu Zhang, Shaohui Yang, Yingjin Song and Jiehua Wang*

\begin{abstract}
Background: Leucine-rich repeat receptor-like kinases (LRR-RLKS) comprise the largest group within the receptorlike kinase (RLK) superfamily in plants. This gene family plays critical and diverse roles in plant growth, development and stress response. Although the LRR-RLK families in Arabidopsis and rice have been previously analyzed, no comprehensive studies have been performed on this gene family in tree species.

Results: In this work, 379 LRR-RLK genes were retrieved from the Populus trichocarpa genome and further grouped into 14 subfamilies based on their structural and sequence similarities. Approximately 82\% (312 out of 379) of the PtLRR-RLK genes are located in segmental duplication blocks indicating the role of duplication process in the expansion of this gene family. The conservation and variation in motif composition and intron/exon arrangement among PtLRR-RLK subfamilies were analyzed to provide additional support for their phylogenetic relationship and more importantly to indicate the potential divergence in their functions. Expression profiling of PtLRR-RLKs showed that they were differentially expressed in different organs and tissues and some PtLRR-RLKs were specifically expressed in meristem tissues, which indicated their potential involvement in tissue development and differentiation. For most AtLRR-RLKs with defined functions, Populus homologues exhibiting similar expression patterns could be identified, which might indicate the functional conservation during evolution. Among 12 types of environmental cues analyzed by the genome-wide microarray data, PtLRR-RLKS showed specific responses to shoot organogenesis, wounding, low ammonium feeding, hypoxia and seasonal dormancy, but not to drought, rewatering after drought, flooding, $\mathrm{AlCl}_{3}$ treatment and bacteria or fungi treatments.

Conclusions: This study provides the first comprehensive genomic analysis of the Populus LRR-RLK gene family. Segmental duplication contributes significantly to the expansion of this gene family. Populus and Arabidopsis LRR$R L K$ homologues not only share similar genetic structures but also exhibit comparable expression patterns which point to the possible functional conservation of these LRR-RLKs in two model systems. Transcriptome profiling provides the first insight into the functional divergence among PtLRR-RLK gene subfamilies and suggests that they might take important roles in growth and adaptation of tree species.
\end{abstract}

Keywords: Populus trichocarpa, Leucine-rich repeat receptor-like kinase (LRR-RLK), Phylogenetic analysis, Motif elicitation, Expression profiling

\footnotetext{
* Correspondence: jiehuawang@tju.edu.cn

School of Environmental Science and Engineering, Tianjin University, Tianjin 300072, China
} 


\section{Background}

Plant cells are able to sense and transduce signals through cell surface receptors which mediate the cell-tocell communication by binding to the extracellular ligands and possessing protein kinase catalytic activities [1]. In 1990, the first plant receptor-like kinase (RLK) was identified in maize [2] and since then, many RLKs have been identified from other plant species. According to the classification based on the extracellular domains, the major group of plant RLK is the leucine-rich repeat RLK family (LRR-RLK) [3]. The structural features of LRR-RLKs include an extracellular receptor domain to perceive signals, a single-pass transmembrane domain to anchor the protein within the membrane and a cytoplasmic serine/threonine (ser/thr) protein kinase domain to transduce the signal downstream via autophosphorylation followed by further phosphorylation of specific substrates $[4,5]$.

Previous reports have classified plant $L R R-R L K$ genes into two broad categories [3]. First, they are important in plant growth and development including morphogenesis, organogenesis and hormone signaling. Second, many LRR-RLKs respond to abiotic and biotic stress and therefore could be defense-related. Some LRR-RLKs have been demonstrated to possess dual functions due to the cross-talk between defense and developmental pathways or due to the recognition of multiple ligands by one signal receptor [6]. For instance, ERECTA is involved in both ovule development and resistance to bacterial wilt $[7,8]$. Although important progress has been made in understanding LRR-RLK functions in recent years, open questions still remain for most LRR-RLKs. The phenotypes associated with various $L R R-R L K \mathrm{mu}-$ tants show that they play roles in diverse processes during growth and development [9]. Meanwhile, the functional redundancy of $L R R-R L K$ family members definitely adds to the complexity of the signaling network they mediate. For example, CLAVATA1 (CLV1) forms a receptor complex with CLV2 upon perception of the CLV3/ESR-related (CLE) peptide derived from CLV3 in the shoot apical meristem to regulate the expression pattern of the stem cell-promoting transcription factor WUSCHEL (WUS) [10-12]. In parallel with CLV1, additional receptors, namely Barely any Meristem (BAM1, BAM2, and BAM3), exhibit similar sequences as CLV1 but perform seemingly contradictory functions. While CLV1 promotes stem cell differentiation, BAM receptors are required for stem cell maintenance [13]. It has been shown that CLV1 and BAM receptors have retained significant similarity in their biochemical function and the differences in their genetic functions appear to be largely driven by their distinctive expression patterns [13].

LRR-RLKs seem to have evolved to acquire novel and diverse functions through neofunctionalization and subfunctionalization by extensive gene duplication [14]. The drastic expansion of this gene family in the land plant lineage is regarded as a plant-specific adaptation for extracellular signal sensing and propagation $[15,16]$. As a forest model organism, poplar is a fast-growing diploid plant that has attracted much attention since its whole genome being sequenced [17]. The structural features and expression profiles of $L R R-R L K$ gene family members have been extensively described in Arabidopsis and rice, however, there has been much less information about this family in woody species including poplar. In the current study, the entire $L R R-R L K$ gene family of Populus trichocarpa was comprehensively identified and analyzed by incorporating sequence phylogeny, gene organization, conserved motif, expression profiling, and gene adaption analysis. Our results provide a framework for further functional investigation on Populus LRR-RLKs and contribute to a better understanding of the complexity of $L R R-R L K$ gene family in higher plants.

\section{Results and discussion \\ Composition and phylogenetic analysis of $L R R-R L K$ gene family in populus trichocarpa}

To date, approximately 213 and $309 L R R-R L K$ genes have been identified in the fully sequenced Arabidopsis and rice genomes, respectively $[18,19]$. In this work, a larger $L R R$ $R L K$ gene family composed of 379 members was identified in the $P$. trichocarpa genome. The number of LRR-RLK genes in Populus is roughly 1.78 fold of that in Arabidopsis, which is consistent with the ratio of putative Populus homologues to each Arabidopsis gene (1.4 1.6) [17]. The detailed information of $L R R-R L K$ family genes in Populus including the accession numbers and the characteristics of the encoded proteins is listed in Additional file 1 and the summarized information concerning each group or subgroup is presented in Table 1. Since the diversity of extracellular domains (ECDs) represents the capability of LRR-RLKs to recognize various ligands and thus constitute the basis of their functional versatility [20], we first identified the ECD for each PtLRR-RLK and constructed the phylogenetic tree to determine their evolutionary relationship (Figure 1, Additional file 2). It has been shown that many events which resulted in the fusion between ECDs and kinase domains occurred early in land plant evolution, thus $R L K$ genes with related kinase sequences tend to have similar ECDs [15,20,21]. In this work, the phylogenetic relationship among the PtLRR-RLKs was also examined based on their catalytic kinase domains and similar categories were obtained (Additional file 3). Since the nodes of the phylogenetic tree based on the ECDs exhibit the best confidence of support, PtLRR-RLKs were classified into 14 subfamilies (I to XIV) accordingly (Figure 1). No well-supported positions could be identified for six PtLRR-RLKs, so they were not included in the 
Table 1 Group and subgroup designations of LRR-RLK proteins from Populus trichocarpa

\begin{tabular}{|c|c|c|c|c|c|c|c|}
\hline Groups & Subgroups & Gene number & PI & $\mathrm{MW}(\mathrm{kDa})$ & aa length & With signal peptide & Homologous Arabidopsis genes based on LRR \\
\hline \multirow[t]{2}{*}{ I } & $\mathrm{a}$ & 16 & $5.29-7.94$ & $43.32-104.52$ & $391-934$ & $56.3 \%$ & FRK1, IOS1, MEE39 \\
\hline & b & 17 & $5.24-8.86$ & $49.58-101.98$ & 455-923 & $58.8 \%$ & FEI1-2, TMKL1 \\
\hline$\|$ & & 29 & $5.19-9.45$ & $68.92-79.48$ & $564-715$ & & NIK1, NIK2, SARK,NIK3, SERK1-5, MRH1 \\
\hline III & & 29 & $5.85-9.19$ & $59.07-74.62$ & $547-684$ & $65.5 \%$ & RLK902, RKL1, RUL1, PRK2A \\
\hline IV & & 20 & $5.43-8.66$ & $59.51-132.35$ & $546-1211$ & & IMK2, IMK3 \\
\hline V & & 11 & $5.82-8.03$ & $67.75-86.62$ & $625-794$ & $54.6 \%$ & SRF1-8, SCM \\
\hline $\mathrm{VI}$ & & 16 & $5.68-9.21$ & $74.95-114.81$ & 673-1072 & & \\
\hline \multirow[t]{2}{*}{ VII } & a & 6 & $5.22-8.72$ & $74.08-133.71$ & $673-1221$ & $83.3 \%$ & \\
\hline & $b$ & 30 & $6.11-8.67$ & $66.14-128.21$ & $599-1162$ & $30.0 \%$ & Populus-specific \\
\hline VIII & & 17 & $5.29-8.16$ & $48.97-106.77$ & $449-978$ & $58.8 \%$ & \\
\hline IX & & 12 & $5.31-6.68$ & $98.97-103.46$ & 908-949 & $100 \%$ & \\
\hline$x$ & & 29 & $5.22-8.77$ & $49.72-130.50$ & 446-1205 & $82.8 \%$ & BIR1, PSKR2, PSKR1,BRL2, BRI1, BRL1,BRL3 \\
\hline \multirow[t]{3}{*}{$X I$} & a & 28 & $5.15-8.03$ & $77.44-113.17$ & $701-1026$ & $85.7 \%$ & HAESA, XIP1, HAIKU2, RLK7, PXY, BAM1-3, CLV1, MOL1 \\
\hline & $b$ & 20 & $5.33-6.06$ & $96.55-136.72$ & $870-1254$ & $55.0 \%$ & GSO1-2, EDA23, ERL1-2, ERECTA \\
\hline & c & 7 & $5.94-8.68$ & $96.63-127.25$ & $887-1145$ & $100.0 \%$ & PEPR1-2 \\
\hline \multirow[t]{2}{*}{ XII } & a & 23 & $5.22-8.56$ & $85.98-126.86$ & 783-1158 & $60.9 \%$ & FLS2, EFR \\
\hline & $b$ & 19 & $5.61-8.44$ & $53.24-115.95$ & 489-1067 & $52.6 \%$ & Populus-specific \\
\hline XIII & & 34 & $5.00-9.14$ & $62.81-135.28$ & $565-1605$ & $47.1 \%$ & MEE62, TOAD1-2 \\
\hline XIV & & 10 & $5.42-8.95$ & $63.75-134.48$ & $574-1237$ & $30.0 \%$ & EMS1 \\
\hline
\end{tabular}

Sequence characteristics of each PtLRR-RLK gene subfamily and its phylogenetic relationship with Arabidopsis LRR-RLKs with known functions are shown. Six PtLRR-RLK proteins did not fit well into clusters. Subgroups that do not include any Arabidopsis LRR-RLK proteins are described as Populus-specific. Information on the AGI code, gene full-name and abbreviation for each AtLRR-RLK gene with defined functions is presented in the Additional file 18.

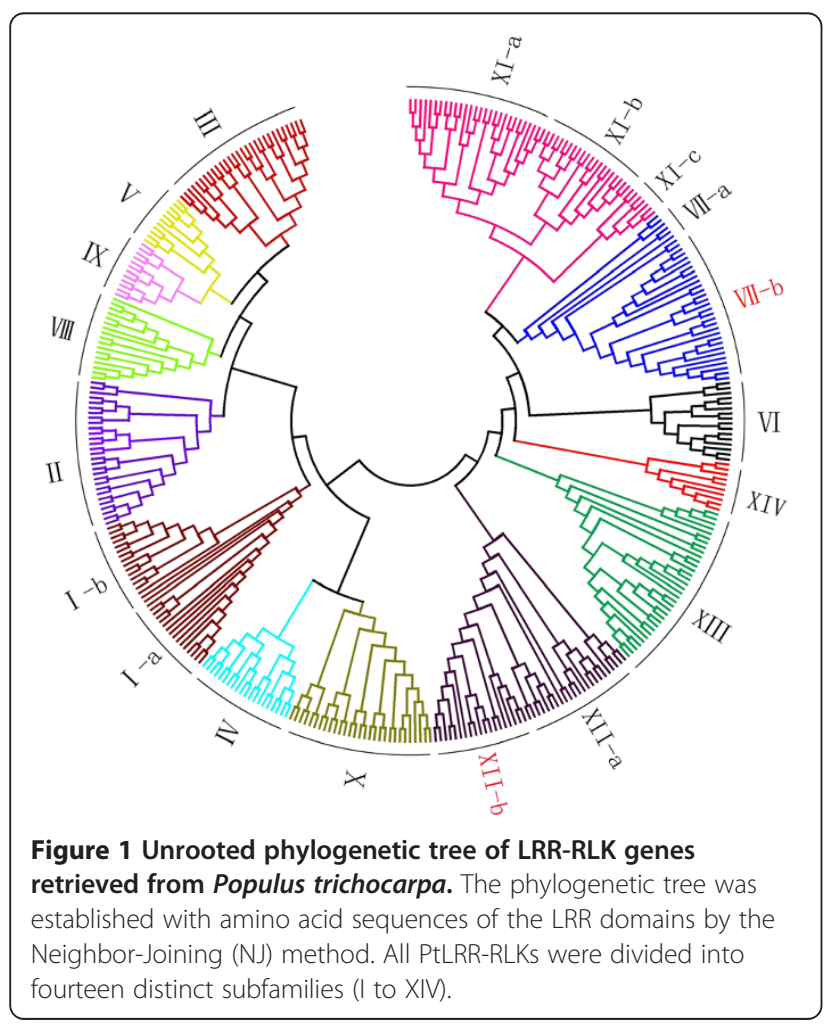

phylogeny (Additional file 1). When PtLRR-RLKs were clustered with AtLRR-RLKs (Additional file 4), the numbering for the Populus LRR-RLK subfamilies was determined based on the nomenclature of the majority of Arabidopsis homologues within the same group. The Populus subfamilies I, II, III and XIII were grouped together with Arabidopsis LRR-RLKs involved in organ/tissue development and with the ones involved in defense signaling. Group IV included only two Arabidopsis Inflorescence Meristem Receptor-like Kinase (IMK) genes which are involved in cell fate specification and proliferation. Group V included the Arabidopsis Strubbelig-receptor Family $(S R F)$ gene family members that affect different aspects of cell wall biology $[22,23]$ and the $S C M$ gene involved in root hair specification [24]. Group VI, VII, VIII and IX had no Arabidopsis orthologs with identified functions. Group X was grouped together with Arabidopsis genes involved in brassinosternoid and peptide signaling such as BAK1-interacting Receptor1 (BIR1), BAK1interacting Receptor-like (BIR-like), and Phytosulfokin receptor1-2 (PSKR1-2) genes [25]. Subgroups XI-a and XI-b were represented with Arabidopsis LRR-RLKs with important roles in organ morphogenesis, cell fate specification and vascular development such as CLV1 and Phloem Intercalated with Xylem (PXY) [26-29], while in the subgroup XI-c, PEP1 receptor1-2 (AtPEPR1-2) is involved in abscisic acid signaling and defense response [30,31]. For 
group XII, subgroup XII-b is Populus specific and subgroup XII-a was clustered with Flagellin-sensitive2 (FLS2) and EF-Tu receptor (EFR) which take part in innate immunity against pathogens [32,33]. Group XIV only included Excess Microsporocytes1 (EMS1) gene, which is involved in endosperm and pollen development [34]. The dispersal pattern of 63 Arabidopsis LRR-RLKs with welldefined roles prompted consideration of the ancestral role of distinct PtLRR-RLK subfamilies and there is a possibility that PtLRR-RLKs belonging to distinct subfamilies perform certain functions in different developmental aspects. For example, the subgroups XI-a and b are more likely to be involved in plant growth and development, while XI-c could be more likely to take roles in plant-microbe interactions. The large size of the Populus LRR-RLK gene family has been regarded as a indication of a great need for LRRRLK genes to participate in more complicated transcriptional regulations in woody species [20]. Meanwhile, the species-specific genes could play important roles in plant responses to a variation of biotic factors, such as the variation of the spectrum of pathogens [35], so it would be very attractive to investigate the functions of the poplarspecific subgroups identified in this work.

\section{Intron-exon organizations of PtLRR-RLKs}

The presence of multiple introns has been shown to be essential for ERECTA expression in Arabidopsis [36], so the intron-exon organizations of PtLRR-RLKs were examined for a clearer understanding of their potential functions. Additional file 5 provides the detailed illustration of the distribution and position of introns for each PtLRR-RLK genes and Figure 2 listed the representative intron/exon structures and their distributions among different gene subfamilies (Figure 2). Out of 379 Populus $L R R-R L K$ genes, 30 had alternative mRNA splicing modes and 25 genes had no intron. One, two, three, four, and five introns was found in 153, 54, 23, 6 and 3 genes, respectively. One hundred and fifteen genes had more than five introns and 72 out of them had more than ten introns (Additional file 5). In terms of exon/intron organization, most of the closely related Populus $L R R-R L K$ genes have roughly the same number and location of introns (Figure 2), which strongly supports their close evolutionary relationship. Populus and Arabidopsis genes belonging to the same subfamilies also exhibit similar genomic features. For example, the gene structure of the Populus subfamily XI were fairly simple and has only one or two introns over their full length sequence, except three genes in the subgroup XI-b which contain as many as 26 introns. Arabidopsis homologues of this group have been shown to play important roles in plant development and organogenesis and most of them contained less than two introns except ERECTA and ERECTA-LIKE1-2 (ERL1-2) which contained as many as
26 introns, this is the most complicated intron/exon structure of AtLRR-RLKs. Although all of the 63 AtLRRRLKs with known functions could be matched with Populus homologues with similar intron/exon structures, the exactly same genetic structures as AtLRR-RLKs were only found in Populus group V, XI and XIII and interestingly, all of them are developmental genes responsible for cell fate specification and morphogenesis (Additional file 6). These results confirmed that the common ancestral genes of PtLRR-RLKs and AtLRR-RLKs already possess multiple intron/exon structures and probably the complicated mRNA processing modes as well. Meanwhile, it seemed possible that the development-related LRR-RLK genes are more conserved in the evolution of genetic structures than the defense-related LRR-RLKs due to their indispensible roles for plant life.

\section{Conserved motifs of PtLRR-RLK genes}

To further reveal the diversification and functional potentials of Populus LRR-RLKs, their conserved motifs were investigated and the consistency of domain arrangement for each subfamily was determined using the Multiple EM for Motif Elicitation (MEME) motif detection software [37]. The LRR motif is usually composed of 20-29 residues with conserved leucines [38] and the consensus residues within the LRR motif were thought to provide a structural skeleton for protein-protein interactions and non-consensus residues within LRRs are though to determine the specificity of such interactions [39]. In total, 17 LRR-related motifs were identified among PtLRR-RLK family members and the basic LRR motif was concluded as LxxLxLxxNx L/f sGx I/l Pxx l/I gxLxx, which shows a good match to the plant LRR consensus LxxLxLxxNxLxGxIPxxLxxLxx and was slightly different from the basic LRR motif in rice (LxxLxLxxNx L/f xGx I/l Pxx 1/i Gx L/c xx) [19]. The most conserved amino acid residues in Populus LRR motifs were Gly at position 1, Pro at position 4, Leu at position 13, 16 and 18, and Asn at position 21. Ile at positions 3 and Leu at position 7 are often substituted by each other and Leu at position 23 is often replaced by Phe (Table 2). Some repeats contain additional conserved residues in other positions, such as a Gly at position 8 of the M23 repeat; Ser residue at position 19 of the M1, M5, M7, M9, M12 and M19 repeat. Since the repetitive structure of LRR makes it capable of the rapid generation of new variants by duplications and deletions of entire repeats [40], the repeat number and distribution of LRR motif have been regarded as important parameters to reflect the evolutionary history. For PtLRR-RLKs, most of the closely related members in the phylogenetic tree kept similar motifs, providing additional support for their phylogenetic relations (Additional file 7). The conserved motifs in the LRR-RLK proteins within the same subfamily may 


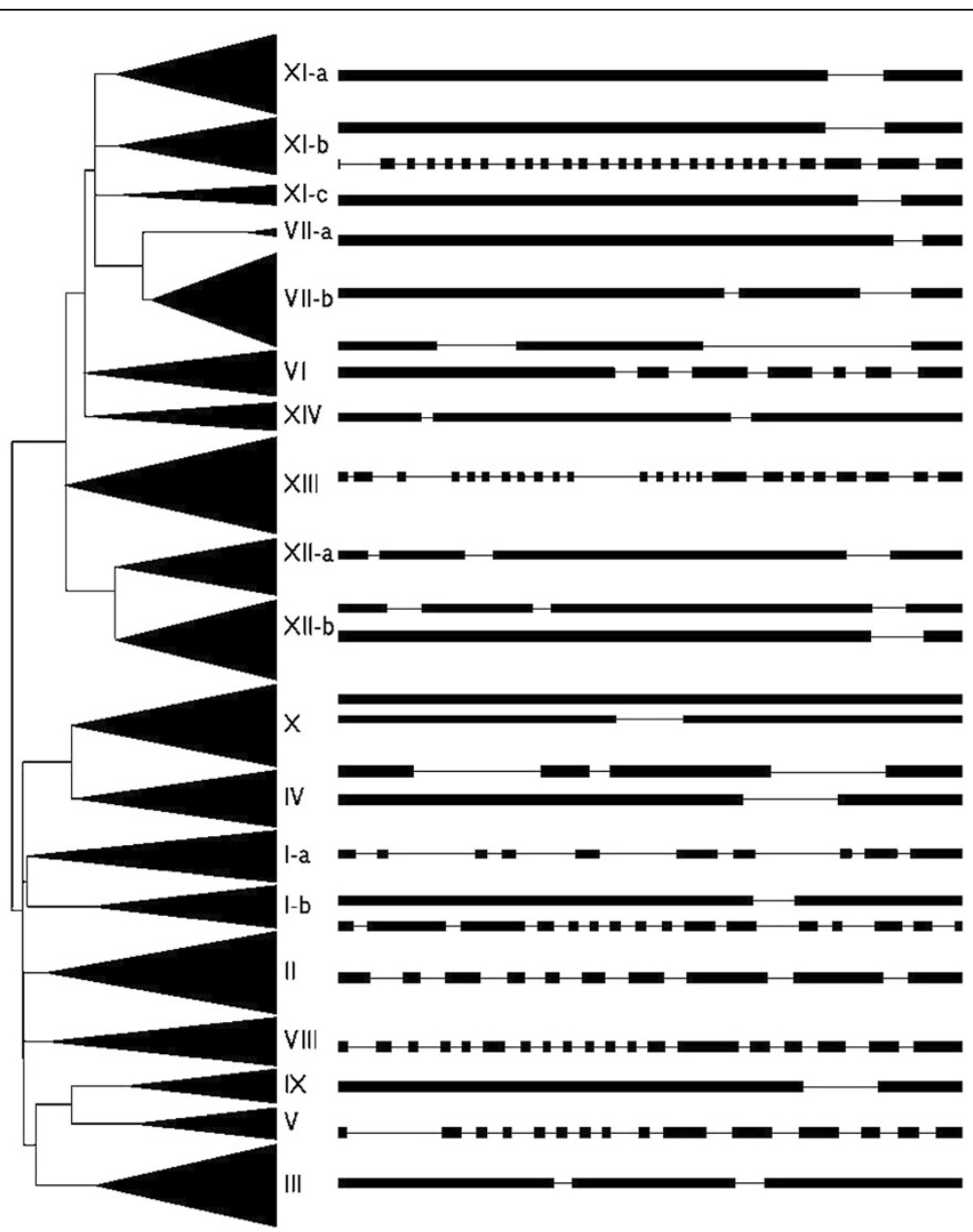

Figure 2 Representative intron-exon structure of each LRR-RLK gene subfamily in Populus. Exons and introns are represented by boxes and lines, respectively; and were drawn to scale with the full encoding regions of their respective gene.

suggest their functional similarities and divergence in motif composition may indicate their functional diversity [35]. Although no group- or subgroup-specific LRR motif was identified, members of different subfamilies did exhibit various degree of complexity in terms of the LRR motif composition (Additional file 7). The most complicated motif composition was observed for the group XI which included all 17 types of LRR motif and in contrast, the group I and II had only 3 to 7 LRR motifs. In addition to the motif composition, the similarity in terms of the arrangement of different LRR motifs also varied among subfamilies (Additional file 7). The arrangement was almost identical for members of subfamily II, III, V, IX and XII-b. The variation in LRR patterns gets more obvious among the members of other subfamilies, although after careful comparison, several clades sharing a regular motif arrangement could still be identified for each subfamily (Additional file 7). The high divergence in the alignments of LRR motif within one subfamily could reflect the functional diversity among their members. In addition to LRR motifs, non-LRR motifs were also identified in the extracellular regions of PtLRR-RLK (Additional file 8). Common motifs including M4, M14 and M17 could be identified in the Nterminal for most of PtLRR-RLKs, while M13 could be found at the C-terminal for most PtLRR-RLKs. Different from these common motifs are certain non-LRR motifs which appear to be subfamily-specific, for example, the motif M24 only appeared in most members of VIII (Additional file 8). PtLRR-RLKs sharing the same or similar motif composition and arrangement could be identified for 50 out of 63 AtLRR-RLKs with known functions (Table 3 and Additional file 9), which supports the theory that the domain organization of most RLK/ Pelle subfamilies was established before the monocotdicot split [16].

When the trans-membrane (TM) domains were predicted by TMHMM, in a total of 379 PtLRR-RLKs, 339 had one TM and 26 PtLRR-RLKs did not have any TM. Further analysis of the remaining 14 PtLRR-RLKs with 
Table 2 Major motifs in the predicted LRR domains of Populus LRR-RLKs

\begin{tabular}{|c|c|c|c|c|c|c|c|c|c|c|c|c|c|c|c|c|c|c|c|c|c|c|c|c|}
\hline LRR motifs & 1 & 2 & 3 & 4 & 5 & 6 & 7 & 8 & 9 & 10 & 11 & 12 & 13 & 14 & 15 & 16 & 17 & 18 & 19 & 20 & 21 & 22 & 23 & 24 \\
\hline M1 & $\underline{\mathbf{G}}$ & $x$ & 1 & $\underline{P}$ & $x$ & $x$ & $\mathrm{~L} / \mathrm{i}$ & G & $x$ & $\mathrm{~L}$ & $x$ & $x$ & $\underline{L}$ & $x$ & $x$ & $\underline{\mathrm{L}}$ & D & $\underline{L}$ & $\mathrm{~S}$ & $x$ & $\underline{N}$ & $x$ & $L / f$ & $s / t$ \\
\hline M2 & $\underline{\mathbf{G}}$ & $x$ & I & $\underline{P}$ & $x$ & $x$ & l/i & G & N & I & $\mathrm{t}$ & $x$ & $\underline{\mathrm{L}}$ & $x$ & $x$ & L & $x$ & L & $x$ & $x$ & $\underline{N}$ & $x$ & $L / f$ & $x$ \\
\hline M3 & $\underline{\mathbf{G}}$ & $x$ & $x$ & $\mathrm{p}$ & $x$ & $x$ & I & $x$ & $x$ & I & $x$ & $x$ & $\underline{\mathrm{L}}$ & $x$ & $x$ & L & $x$ & L & s & $x$ & $\underline{N}$ & $x$ & $F / l$ & $s / t$ \\
\hline M5 & G & $x$ & $1 / 1$ & $\mathbf{P}$ & $x$ & $x$ & I & $\mathrm{g}$ & $x$ & L & $x$ & $x$ & L & $x$ & $x$ & L & $D$ & L & S & $x$ & $\underline{N}$ & $x$ & L & $t$ \\
\hline M6 & G & $x$ & I & $\mathbf{P}$ & $x$ & $x$ & $\mathrm{~L} / \mathrm{i}$ & $x$ & $x$ & C & $x$ & $x$ & $\underline{\mathrm{L}}$ & $x$ & $x$ & L & $x$ & L & $x$ & $x$ & $\underline{N}$ & $x$ & $L / f$ & $\mathrm{~s}$ \\
\hline M7 & $\underline{\mathbf{G}}$ & $p$ & $|/| / V$ & $\underline{\mathbf{P}}$ & $x$ & $x$ & I & $x$ & $x$ & I & $x$ & $x$ & L & $x$ & $x$ & L & $D$ & L & $S$ & $x$ & $\underline{\mathbf{N}}$ & $x$ & $L / f$ & $S$ \\
\hline M8 & G & $x$ & I & $P$ & $x$ & e & $1 / \mathrm{i}$ & G & $x$ & L & $x$ & $x$ & $\underline{\mathbf{L}}$ & $x$ & $x$ & L & $x$ & L & $x$ & $x$ & $\underline{\mathbf{N}}$ & $x$ & L & $x$ \\
\hline M9 & $\underline{\mathbf{G}}$ & $x$ & $|/|$ & $\underline{\mathbf{P}}$ & $x$ & $x$ & I/f/i & g & $n$ & $\mathrm{~L}$ & s & $n$ & $\underline{\mathbf{L}}$ & $x$ & $x$ & L & $d$ & L & $S$ & $x$ & $\underline{\mathbf{N}}$ & $x$ & $L / f$ & $x$ \\
\hline M10 & $\underline{\mathbf{G}}$ & $x$ & $1 / \mid$ & $\underline{\mathbf{P}}$ & $x$ & $x$ & I/f/i & $\mathrm{g}$ & $N$ & $\mathrm{~L}$ & $x$ & $n$ & $\underline{\mathbf{L}}$ & $x$ & $x$ & L & $x$ & L & $\mathrm{s}$ & $x$ & $\underline{\mathbf{N}}$ & $x$ & $L / f$ & s \\
\hline M11 & $\underline{\mathbf{G}}$ & $x$ & i & $p$ & $x$ & $\mathrm{~s}$ & I & g & $x$ & 1 & $x$ & $n$ & $\underline{\mathbf{L}}$ & $x$ & $x$ & L & $x$ & L & $\mathrm{s}$ & $x$ & $\underline{\mathbf{N}}$ & $x$ & $L / f$ & s \\
\hline $\mathrm{M} 12$ & G & $x$ & $x$ & $p$ & $x$ & $x$ & I/f/i & $x$ & $x$ & I & $x$ & $n$ & $\underline{\mathbf{L}}$ & $x$ & $y$ & L & $d$ & L & $S$ & $x$ & $\underline{\mathbf{N}}$ & $x$ & $\mathrm{I} / \mathrm{F}$ & $x$ \\
\hline M15 & $\underline{\mathbf{G}}$ & $x$ & i & $P$ & $x$ & $x$ & 1 & $x$ & $x$ & I & $x$ & $x$ & $\underline{\mathbf{L}}$ & $x$ & $x$ & $L$ & $d$ & L & S & $x$ & $\underline{\mathbf{N}}$ & $x$ & $L / f$ & S \\
\hline M16 & G & $x$ & $1 / \mid$ & $P$ & $x$ & $x$ & I/i & $\mathrm{g}$ & $x$ & $\mathrm{~L}$ & $x$ & $x$ & $\underline{\mathbf{L}}$ & $x$ & $x$ & L & $x$ & $L$ & S & $x$ & $\mathbf{N}$ & $x$ & $L / f$ & $x$ \\
\hline M18 & G & s & $i / / / f$ & $\mathbf{P}$ & $x$ & $x$ & $\mathrm{~L} / \mathrm{i}$ & g & $N$ & $\mathrm{~L}$ & $x$ & $x$ & $\underline{\mathbf{L}}$ & $x$ & $x$ & $L$ & $x$ & $L$ & $x$ & $x$ & $N$ & $x$ & $L / f$ & $x$ \\
\hline M19 & $\underline{\mathbf{G}}$ & $x$ & $|/|$ & $\underline{\mathbf{P}}$ & $x$ & s/e/a & $l / f$ & $x$ & $x$ & 1 & $x$ & $x$ & $\mathbf{L}$ & $x$ & $x$ & L & $d$ & $L$ & $S$ & $x$ & $\underline{\mathbf{N}}$ & $x$ & $\mathrm{~L} / \mathrm{f}$ & $\mathrm{s}$ \\
\hline M20 & $\underline{\mathbf{G}}$ & $x$ & $\mathrm{i} / \mathrm{l}$ & $P$ & $x$ & $x$ & l/i & $x$ & $x$ & I & $x$ & $n$ & $\underline{\mathbf{L}}$ & $x$ & $x$ & L & $x$ & $L$ & $x$ & $x$ & $\underline{\mathbf{N}}$ & $x$ & I/F & s \\
\hline M23 & $\underline{\mathbf{G}}$ & $x$ & $1 / /$ & $\underline{P}$ & $x$ & e & $\mathrm{L} / \mathrm{i}$ & G & $n$ & L & $x$ & $x$ & L & $x$ & $x$ & L & $x$ & I & $x$ & $x$ & $\underline{\mathbf{N}}$ & $x$ & $L / f$ & $\mathrm{~s} / \mathrm{t}$ \\
\hline
\end{tabular}

If the bits value of the amino acid at this position is smaller than 0.5 , it is represented with $x ; 1>$ bits $\geq 0.5$, with lowercase; $2>$ Bits $\geq 1$, with capital letter; $3>$ bits $\geq$ 2 , with bold capital; bits $\geq 3$, with underlined capital letter in bold.

two TMs revealed one of them is atypical. The RLK domain of most PtLRR-RLK consists of approximately 250-280 amino acid residues with a maximum of 324 and a minimum of 168. In literature, plant RLK could be divided into 12 conserved subdomains (I-XII) from Nto C-terminal [41]. In the 2- lobed structure of the RLK domain, the smaller lobe is composed of subdomains I to IV and is involved in anchoring and orienting the nucleotide. The larger lobe is composed of subdomain VI to XI and is largely responsible for binding the peptide substrate and initiating phosphor-transfer [41]. In the kinase part of all PtLRR-RLKs, 25 motifs are identified which are similar to those identified for rice LRR-RLKs and were named as 1 to 25 according to the frequencies of their appearance (Table 4). Although most motifs did not seem to be subfamily-specific, motif 10,13 and 16 only appeared in the subgroup VII and motif 15, 24 and 25 only showed up in the subfamily XII. Since only these two subfamilies included a Populus-specific clade in the phylogenic tree, these specific motifs may, to some extent, attribute to the functional divergence of these subfamily members in poplar.

Kinase is commonly referred to as arginine-aspartate (RD) kinases if it is strongly activated by the phosphorylation of the activation loop and they usually contain an $\operatorname{Arg}(\mathrm{R})$ in the subdomain preceding the catalytic loop [41]. Conversely, a smaller number of kinases are referred to as Non-arginine-aspartate (non-RD) kinases which lack the conserved $\mathrm{R}$ in subdomain VI $[42,43]$. It has been proposed that the signal of pathogen recognition mediated by RLKs is usually through a non-RD kinase [44]. In PtLRR-RLKs, about half are RD-kinases including all the members of subfamily VII and IX. Interestingly, no Arabidopsis homolog with known functions has been identified for these two subfamilies and the VII-b subfamily is Populus-specific. In contrast, all members in the subfamily III, IV, V and XII are non-RD kinases although the Arabidopsis LRR-RLKs grouped with them take part in both defense and development (Table 1).

\section{Contributions of tandem and large-scale duplications to the family size of PtLRR-RLKs}

The explosion of members of a gene family has generally occurred as the result of repetitive tandem duplication (TD) and segmental and/or whole genome duplication events (S/WGD). PtLRR-RLK genes were comprehensively distributed within the poplar genome and 22 genes are localized to unassembled genomic sequence scaffolds and thus could not be mapped to any particular chromosome (Figure 3). Approximately 82\% (312 out of 379) PtLRR-RLK genes are located in the replicated region, which is different from rice and Arabidopsis in which the frequencies of genes generated by S/WGDs are much lower (11\% in rice and 26\% in Arabidopsis) [45]. Among them, 140 genes lacked duplicates on the corresponding duplicated blocks, suggesting that dynamic rearrangement, mutation or segmental loss may have 
Table 3 Arabidopsis and Poplus LRR-RLK genes resembling each other in motif configuration

\begin{tabular}{|c|c|c|c|}
\hline At Gene Family & Gene name & At Gene locus & Pt Gene locus \\
\hline \multirow[t]{13}{*}{$\|$} & & & POPTR_0004s10790.1 \\
\hline & NIK1 & At5G16000 & POPTR_0017s14360.1 \\
\hline & NIK2 & At3G25560 & POPTR_0008s10970.1 \\
\hline & NIK3 & At1G60800 & POPTR_0010s14410.1 \\
\hline & & & POPTR_0008s19310.1 \\
\hline & SARK & At4G30520 & POPTR_0006s19320.1 \\
\hline & & & POPTR_0018s11030.1 \\
\hline & SERK3 & At4G33430 & POPTR_0001s21420.1 \\
\hline & SERK4 & At2G13790 & POPTR_0003s01740.1 \\
\hline & SERK5 & At2G13800 & \\
\hline & SERK1 & At1G71830 & POPTR_0005s08500.1 \\
\hline & SERK2 & At1G34210 & POPTR_0013s12150.1 \\
\hline & & & POPTR_0019s11740.1 \\
\hline \multirow[t]{8}{*}{ III } & PRK2A & At2G07040 & POPTR_0018s14390.1 \\
\hline & & & POPTR_0006s07820.1 \\
\hline & RUL1 & At5G05160.1 & POPTR_0019s15210.1 \\
\hline & & & POPTR_0013s15480.1 \\
\hline & RLK902 & At3G17840 & POPTR_0012s04170.1 \\
\hline & RKL1 & At1G48480 & POPTR_0015s04920.1 \\
\hline & LRR1 & At5G16590 & \\
\hline & TMKL1 & At3G24660 & POPTR_0002s25300.1 \\
\hline \multirow[t]{11}{*}{ V } & & & POPTR_0001s16170.1 \\
\hline & SRF7 & AT3G14350 & POPTR_0003s07130.1 \\
\hline & SRF6 & AT1G53730 & POPTR_0004s00710.1 \\
\hline & SRF8 & AT4G22130 & POPTR_0011s01540.1 \\
\hline & SRF4 & AT3G13065 & POPTR_0007s15170.1 \\
\hline & SRF5 & AT1G78980 & POPTR_0014s00340.1 \\
\hline & SRF2 & AT5G06820 & POPTR_0006s20510.1 \\
\hline & SCM & AT1G11130 & POPTR_0004s03720.1 \\
\hline & SRF3 & AT4G03390 & POPTR_0011s04540.1 \\
\hline & SRF1 & AT2G20850 & POPTR_0019s13490.1 \\
\hline & & & POPTR_0013s14080.1 \\
\hline \multirow[t]{2}{*}{ VI-b } & $\mathrm{MRH} 1$ & AT4G18640 & POPTR_0011s07250.1 \\
\hline & & & POPTR_0004s05680.1 \\
\hline \multirow[t]{5}{*}{$X-a$} & & & POPTR_0008s07830.1 \\
\hline & & & POPTR_0002s24100.1 \\
\hline & BIR1 & AT5G48380 & POPTR_0017s03710.1 \\
\hline & & & POPTR_0010s18540.1 \\
\hline & & & POPTR_0010s18550.1 \\
\hline \multirow[t]{4}{*}{$x-b$} & BRI1 & At4G39400 & POPTR_0007s06940.1 \\
\hline & BRL1 & At1G55610 & POPTR_0001s47680.1 \\
\hline & BRL3 & At3G13380 & POPTR_0011s17240.1 \\
\hline & PSKR1 & AT2G02220 & POPTR_0008s14390.1 \\
\hline
\end{tabular}

Table 3 Arabidopsis and Poplus LRR-RLK genes resembling each other in motif configuration (Continued)

\begin{tabular}{|c|c|c|c|}
\hline & & & POPTR_0010s10790.1 \\
\hline & PSKR2 & AT5G53890 & POPTR_0011s11780.1 \\
\hline \multirow[t]{17}{*}{$X I$} & $\mathrm{HAE}$ & At4G28490 & POPTR_0007s01340.1 \\
\hline & & & POPTR_0017s04790.1 \\
\hline & XIP1 & At5G49660 & POPTR_0002s11230.1 \\
\hline & RLK7 & At1G09970 & POPTR_0002s10700.1 \\
\hline & HAIKU2 & At3G19700 & POPTR_0009s08540.1 \\
\hline & MOL1 & At5G51350 & POPTR_0001s00820.1 \\
\hline & PXY & At5G61480 & POPTR_0003s10680.1 \\
\hline & CLV1 & At1G75820 & POPTR_0002s02140.1 \\
\hline & & & POPTR_0005s26300.1 \\
\hline & BAM1 & At5G65700 & POPTR_0007s14500.1 \\
\hline & BAM2 & At3G49670 & \\
\hline & BAM3 & At4G20270 & POPTR_0001s12420.1 \\
\hline & & & POPTR_0003s15600.1 \\
\hline & GSO1 & At4G20140 & POPTR_0001s12290.1 \\
\hline & EDA23 & At5G44700 & \\
\hline & PEPR2 & At1G17750 & POPTR_0008s00810.1 \\
\hline & PEPR1 & At1G73080 & POPTR_0008s00970.1 \\
\hline \multirow[t]{2}{*}{ XIII-a } & FEl1 & AT1G31420 & POPTR_0001s01120.1 \\
\hline & FEl1 & AT2G35620 & POPTR_0012s07290.1 \\
\hline \multirow[t]{3}{*}{$X \mid I-b$} & ERL1 & At5G62230 & POPTR_0015s14270.1 \\
\hline & ERL2 & At5G07180 & POPTR_0012s14250.1 \\
\hline & ERECTA & At2G26330 & POPTR_0006s23680.1 \\
\hline
\end{tabular}

occurred following the segmental duplication. According to previous literature, a chromosome region containing two or more genes within $200 \mathrm{~kb}$ can be defined as a gene cluster [46]. In poplar, 72 PtLRR-RLK genes were located in 20 tandem duplication clusters (Figure 3). The smallest tandem duplication clusters consisted of only 2 genes and the largest cluster had 8 tightly linked genes on chromosome 15 and 19. The clusters were distributed unevenly among the 14 phylogenetic groups, and Populus-specific subgroup VII-b contains 6 clusters incorporating $86.7 \%$ of the genes of this subgroup. By contrast, group II, III, IV, V, VI, IX had no clusters present (Additional file 10).

\section{Differential expression profiles of populus $L R R-R L K$ genes}

To gain a broader understanding of the function of LRR-RLKs, we analyzed the divergence among Populus $L R R-R L K$ genes in spatial and temporal expression and expression in response to specific environmental signals. Probe sets were readily identifiable for 283 out of 379 PtLRR-RLKs in the PopGenExpress data set, and their distinct transcript abundance patterns were retrieved by 
Table 4 Major motifs in the predicted protein kinase domains of Populus LRR-RLKs

\begin{tabular}{|c|c|c|}
\hline Motif & Sequence & Corresponding motif sequence in rice RLK \\
\hline 20 & $\underline{\mathrm{FN}} / \mathrm{DSN} / \mathrm{KYC}$ & \\
\hline 6 & $\mathrm{I} / \mathbf{G} \mathbf{\mathbf { G }} \underline{\mathbf{G}} \mathrm{G} \underline{\mathbf{G}} \times \mathbf{V} \underline{\mathbf{Y} K} / \mathrm{rA} / \mathrm{G} \times \mathrm{L} / \mathrm{mx}$ & 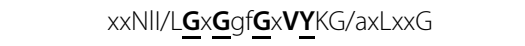 \\
\hline 9 & $\mathrm{~d} \mathbf{G} \times \times \vee \underline{\mathbf{A V K K}} K / \mathrm{rL}$ & $x \mathbf{V} \underline{\mathbf{A V}} / \underline{\mathrm{i}} \underline{\mathrm{K} V L X X X X}$ \\
\hline 5 & 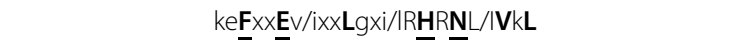 & xs $\underline{F} \times x \times E C / v e x L / i s x V / i R \underline{H} R \underline{N} L / i V x L / i x G / t x C x x x d$ \\
\hline 11 & $y \underline{G} \underline{G} \underline{S} x \times k x x F$ & \\
\hline 23 & $\underline{\mathbf{E}} \mathrm{A} / \mathrm{d} S / T \times Q / n L \underline{\mathbf{S}} \times \mathrm{NQ} / \mathrm{pS} / \mathrm{TS} \underline{\mathbf{S}} \mid \mathrm{NG}$ & \\
\hline 19 & RAY/fYy/wskDEKL & \\
\hline 15 & $\underline{\mathrm{TA}} / \mathrm{s} \underline{\mathrm{CS}} \mathrm{S} / \mathrm{GV} / \underline{\mathrm{D}} \mathrm{F} / \mathrm{yQ} / \mathrm{kGNDFKA}$ & $x x \underline{G} \cap D / e \underline{F K A}$ \\
\hline 4 & 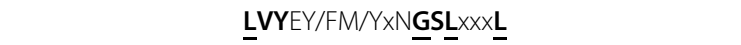 & 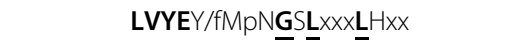 \\
\hline 14 & xexxxxxLDW & \\
\hline 3 & $\times \underline{\mathbf{R}} \mid \mathrm{k} / \mathrm{nI} / \mathrm{VAxG} / \mathrm{dV} / \mathrm{a} \underline{\mathbf{A}} \times \mathrm{G} / \mathrm{AL} \times \mathrm{Y} \underline{\mathbf{L}} \mathbf{H}$ & $\mathrm{LdW} /|\times \times \underline{\mathbf{R}}| \times \times \mathbf{A} \mid \mathrm{G} / \mathrm{DvvA \times G/A \times YL} \underline{\mathbf{H}}$ \\
\hline 1 & 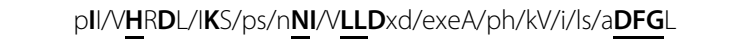 & $\mathrm{xl} / \mathrm{vv} / \mathrm{iH} \underline{\mathrm{H}} / \mathrm{CD} \mid / / / \mathrm{VK} \mathrm{s} / \mathrm{pS} \underline{\mathbf{N}} \mathrm{I} / \mathrm{VLLD}$ \\
\hline 18 & $\mathrm{~L} \times x \times x d T / S \underline{H} V / / T / S \underline{T}$ & \\
\hline 13 & LLMPDSSNWTS/a & \\
\hline 25 & $\underline{\mathbf{E}} \mathrm{A} / \mathrm{dS} / \mathrm{T} \times \mathrm{q} / \mathrm{h} / \mathrm{hl} / \mathrm{h} \underline{\mathbf{S}} \times \mathbf{N} \mathrm{Q} / \mathrm{pS} / \mathrm{T} \underline{\mathbf{S}} \mathbf{S} / \mathrm{VG}$ & xssxsGSt/sxxefsxqxExxP \\
\hline 7 & xAGT/SX $\underline{\mathbf{G Y}} \times \mathbf{A P E Y a x T}$ & 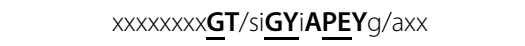 \\
\hline 22 & xxxxxxxxxg$\underline{\mathbf{Y}} / \mathrm{kA} / \mathrm{s}$ PExxxxkK/r & $x x x x x x$ GYRAPE $v x x x x x / r S / t x$ \\
\hline 2 & $\mathrm{eK} \times \underline{\mathbf{D V Y}} \mathbf{S F} / \mathbf{y} \underline{\mathbf{G}} / \mathrm{iV} / \mid \mathbf{L L} / \mathrm{m} \underline{\mathbf{E}} \mathrm{L} / \mathbf{T} / \mathrm{s} \underline{\mathbf{G}} \mathrm{K} / \mathrm{rXP}$ & 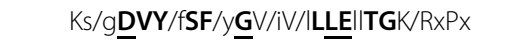 \\
\hline 12 & xxxdLivx吕/a & $x \mathbf{L} v x \underline{\mathbf{W}} \vee / a x x x x x x x x x x$ \\
\hline 16 & $\underline{\mathrm{GD}} \mathrm{L} / \mathrm{F} \underline{\mathrm{ISS}} / \mathrm{A} \underline{\mathrm{L} L} / \mathrm{M} / \mathrm{SSS} / \mathrm{PA} / \mathrm{GSS} / \mathrm{TS}$ & \\
\hline 17 & 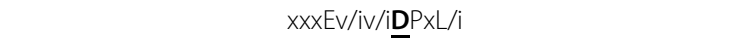 & $\mathrm{xev} / \mathrm{iv} / \mathbf{I} \underline{\mathrm{D}} \mathrm{p} \mathrm{L} \mathrm{xxx}$ \\
\hline 21 & 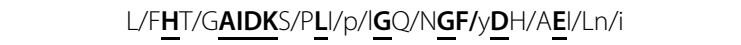 & \\
\hline 10 & 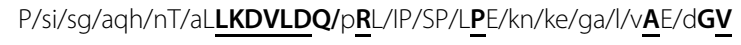 & \\
\hline 24 & xxsh/fg/egNSI/es/exn/eK/RV/IECLV/ISI & \\
\hline 8 & 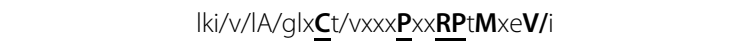 & $|x \times v| x \mid / v / i A / g L x \underline{G} t \times x \times \mathbf{P} \times \underline{\mathbf{R} P} \times \mathbf{M} \times e$ \\
\hline
\end{tabular}

If the bits value of the amino acid at this position is smaller than 0.5 , it is represented with $x ; 1>$ bits $\geq 0.5$, with lowercase; $2>$ bits $\geq 1$, with capital letter; $3>$ bits $\geq 2$, with bold capital; bits $>3$, with underlined capital letter in bold.

the Populus Electronic Fluorescent Pictograph (eFP) browser [47]. Most Populus LRR-RLK genes demonstrated distinct tissue specific expression patterns except for mature leaves, where all have low transcriptional levels (Additional file 11). Filtering was added to select genes that had at least a 2-fold higher expression in one specific tissue compared to the median expression level of all analyzed tissues. Out of the PtLRR-RLK genes for which microarray data are available, 28\%, 29\%, 15\%, 27\% and $19 \%$ showed specific transcript accumulations in young leaf, roots, female catkins, male catkins and developing xylem, respectively (Figure 4A). Identification of the genes predominantly expressed in meristem tissues provides an important clue for their functions during cell fate specification and organ formation. Therefore, the expression of PtLRR-RLKs in multiple meristem tissues was investigated which may provide a further solid basis to select meristem-specific genes for related functional validation (Figure 4B).
Out of 16 tandem duplicated gene clusters, 8 clusters exhibited similar expression patterns among genes with expression data available (Additional file 12). It has been reported that in both rice and Arabidopsis, more than $50 \%$ of duplicate LRR-RLK gene pairs that were generated by a whole genome duplication event exhibited expressional divergence $[48,49]$. In poplar, among 82 pairs of $L R R-R L K$ paralogs with expression data available, 68 (group I), 10 (group II) and 4 (group III) pairs shared $>80 \%, 60-80 \%$ and $<60 \%$ similarities over their full amino acid sequences, respectively. When expression patterns were compared, $70 \%, 30 \%$ and $0 \%$ pairs shared similar expression pattern in group I, II and III, respectively (Additional file 13). Thus, the expressional diversity of duplicated genes in poplar was correlated with the sequence variation which may represent a dynamic functional diversification of this gene family over evolutionary time and contribute to the adaptability of trees. Among the 63 Arabidopsis LRR-RLKs with known 


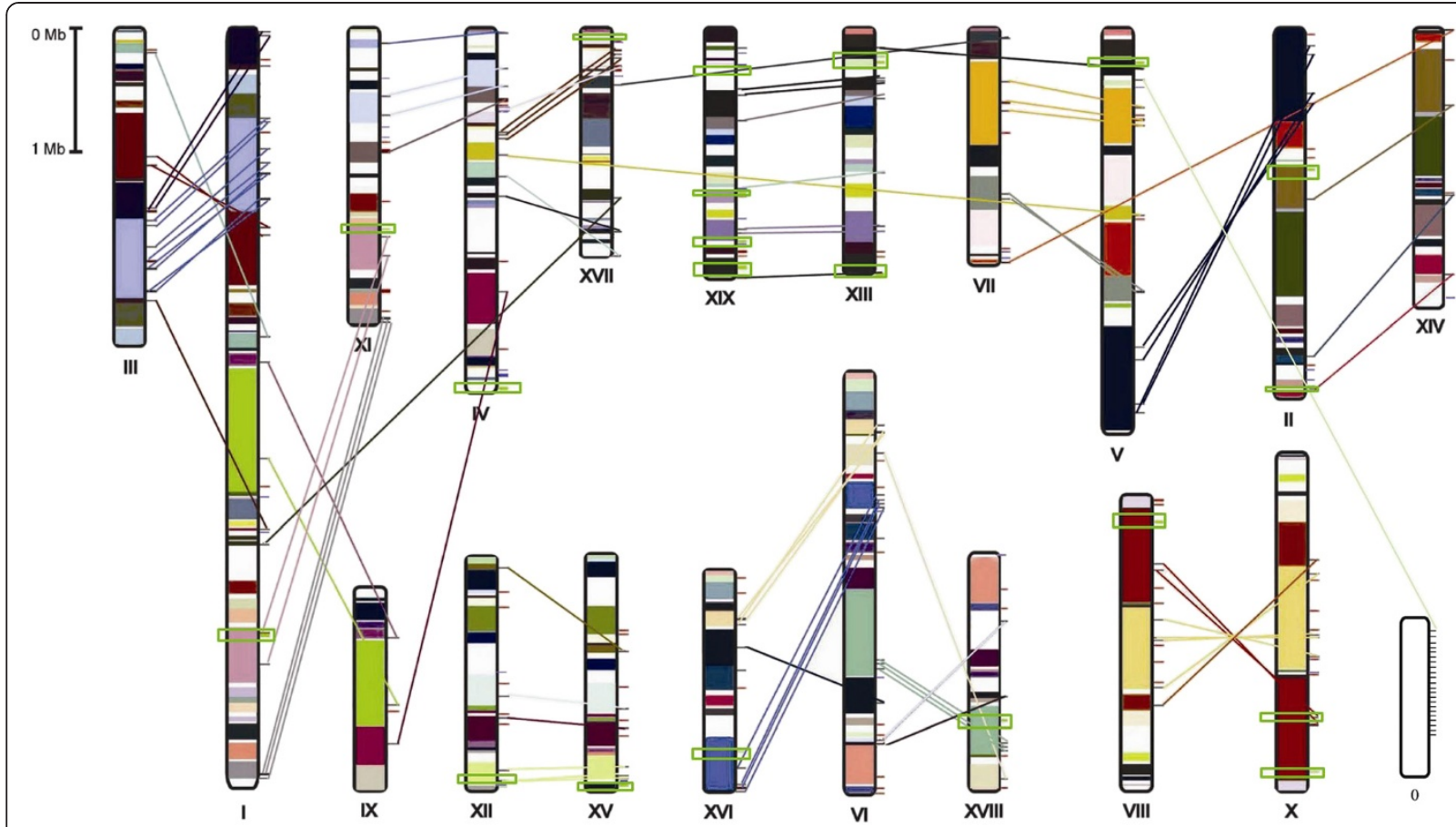

Figure 3 Distribution of LRR-RLK genes on Populus chromosomes. Genes are mapped to LG according the Joint Genome Institute Poplar Genome version 2.0. The schematic diagram of Populus chromosome organization arisen from the salicoid genome duplication event was adapted from Tuskan et al., (2006) [17]. Scale represents a 1 Mb chromosomal distance. Segmental duplicated homologous blocks are indicated with the same color and corresponding sister gene pairs were connected by colored lines. Tandemly duplicated genes are encompassed in the green boxes.

functions, 52 genes showed obvious tissue-specific expression instead of a whole-plant expression as illustrated by eFP and for 45 genes, their Populus homologues showed a similar spatial expression pattern (Additional file 14). This result supports that orthologous genes from different species may retain similar temporal and spatial expression patterns [50,51].

By complete searching of the digital expression profiles from the Gene Expression Omnibus (GEO) repository at NCBI website, we also investigated the expression patterns of the PtLRR-RLK genes during shoot organogenesis and in response to various stress stimulus including drought, cold, hypoxia, nitrogen limitation, aluminum stress in roots, bacteria, fungi and mimic wounding (Additional file 15). In a total of 12 treatments, the expression profile of PtLRR-RLKs varied considerably when exposed to 7 treatments, except for infection by Marssonina pathogen and Melampsora rust fungi, drought and aluminum stress in roots. Genes responsive to various treatments were summarized as heat maps in Additional file 16. The percentages of members of each subfamily being induced or suppressed for each treatment were listed in Additional file 17 and summarized in the format of heatmap in Figure 5. It can be seen that LRR-RLKs respond to various stimulates in a temporal and spatial manner by changing the expression profiles of different gene sets. For example, in wounding experiment, 90 hours after treatment (GSE16785), 102 and 59 PtLRR-RLK genes were upregulated in leaf LPI5 and root, respectively, and qualitative differences in the induction patterns were detected for these two types of tissue (Figure 5). When the sampling time was extended to one week (GSE16783), only 31 and 87 genes were detected as induced in LPI1 leaves and LPI5 leaves, respectively, and compared to very young leaves (LPI1), the older leaves with LPI5 were much more enriched with up-regulated LRR-RLKs, which were overrepresented by members from subfamily III, IV, V, IX and XI-b (Figure 5). In another assay, the gene expression response of Populus tremuloides cell suspension cultures to methyl jasmonate feeding was analyzed; the transcript level of 37 PtLRR-RLKS was elevated. All these data indicated that LRR-RLK gene family plays an indispensable role in wounding defense of tree species. When confronted with ammonium shortage, at a 4-week checkpoint, the induction was more dramatic in young leaves (LPI2) than the older leaves (LPI5). With the progression of the ammonium shortage, the transcript of LRR-RLKs from subfamily III, IV, IX and XI-b got obviously repressed in the older leaves (Figure 5). When the effect of hypoxia on gene expression was investigated in grey poplar, 117 genes responded by induction in leaves with only 11 genes got induced in roots. This located induction 


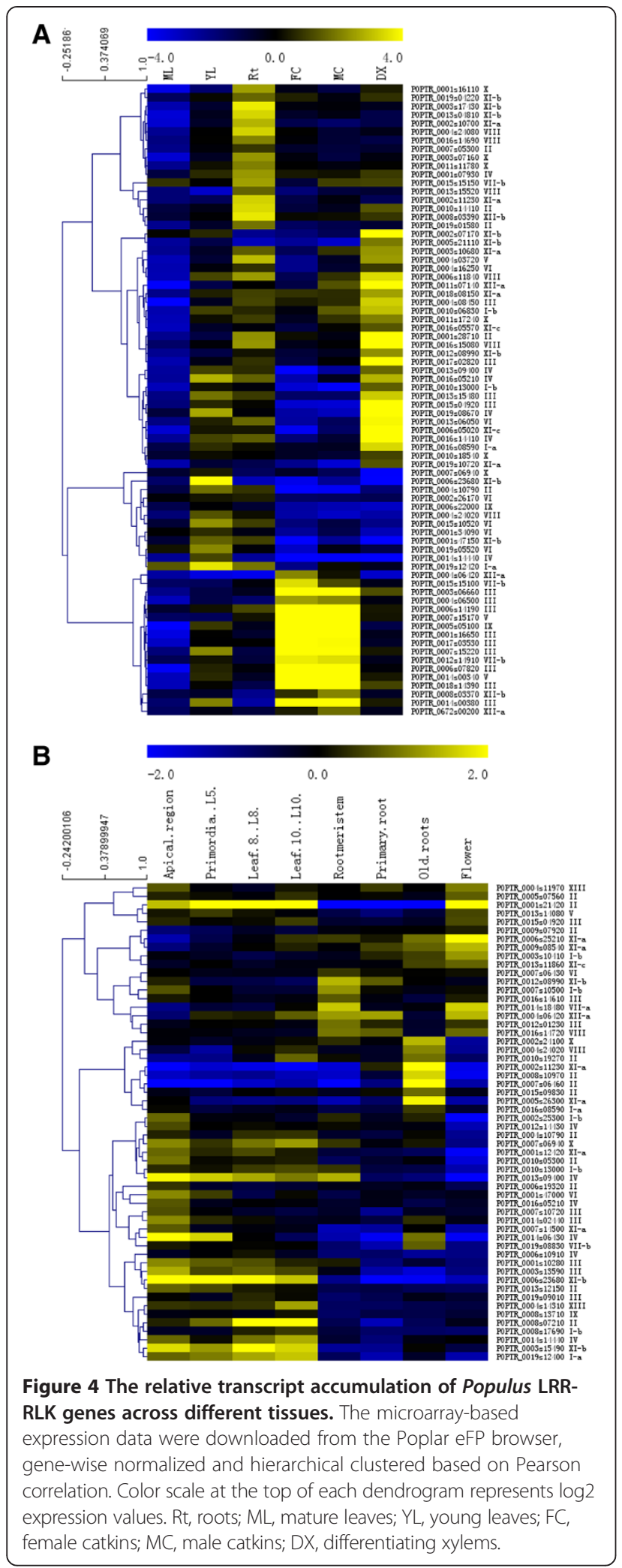

pattern may imply the localized functions of different PtLRR-RLK members. From Figure 5, it was seen that members of different LRR-RLK subfamilies act in an overlapping manner when dealing with different stimulus which indicated that cross talk and signal integration exist among different signaling pathways mediated by PtLRRRLKs. In terms of down-regulation, several things need to be pointed out (Figure 5B). First, members of VII and XIV subfamily were highly repressed in LPI5 leaves one week after wounding. Second, only 26 PtLRR-RLK genes got transcriptionally induced in the winter survival and maintenance mechanism of $P$. trichocarpa, 144 genes responded with repression instead (GSE21480). Third, in the hypoxia treatment, with more than $90 \%$ of the induced genes was located in the leaf tissue, $88 \%$ down-regulated genes were found in the root tissue instead. In summary, although it is hard to assign distinct roles to different Populus LRR-RLK subfamilies based on the results of limited microarray analysis, it could be reasonable to suggest that PtLRR-RLKs are widely involved in different aspects of plant development in both normal and stressed circumstances. However, subset of Arabidopsis LRR-RLK genes have previously been shown to play crucial roles in biotic stress response (Additional file 18). Two biotic signals, Marssonina pathogen and Melampsora rust fungi, did not cause significant change of gene expression profiles in the current study, which indicates a need for more microarray experiments to better understand the roles of Populus $L R R-R L K$ genes in biotic defense. For trees, it is unlikely to generate a collection of LRR-RLK T-DNA insertion mutants, as in Arabidopsis, to be easily applied for the analysis of other developmental aspects. The results from this study could provide insights into possible functions for some PtLRR-RLKs before future functional analyses would eventually elucidate their biological meanings.

\section{Conclusions}

Characterization of LRR-RLK genes in a ligneous species would facilitate a better understanding of the evolutionary processes and functions of this gene family. The current work shows that the LRR-RLKs represent a large gene family in Populus trichocarpa. Gene structures, motif composition and arrangements are considerably conserved among the (sub)groups. The distribution of genes was found to be non-random across chromosomes and a high proportion of the genes are located in segmental duplicated regions instead of tandem duplicated clusters. For most of the 63 Arabidopsis with known functions, Populus homologues always could be identified with similar genetic structure, motif character and expression profiles, providing insight into the evolutionary and functional conservation of this gene family in plant species. Expression patterns based on microarray data suggest that many PtLRR-RLK genes are expressed 


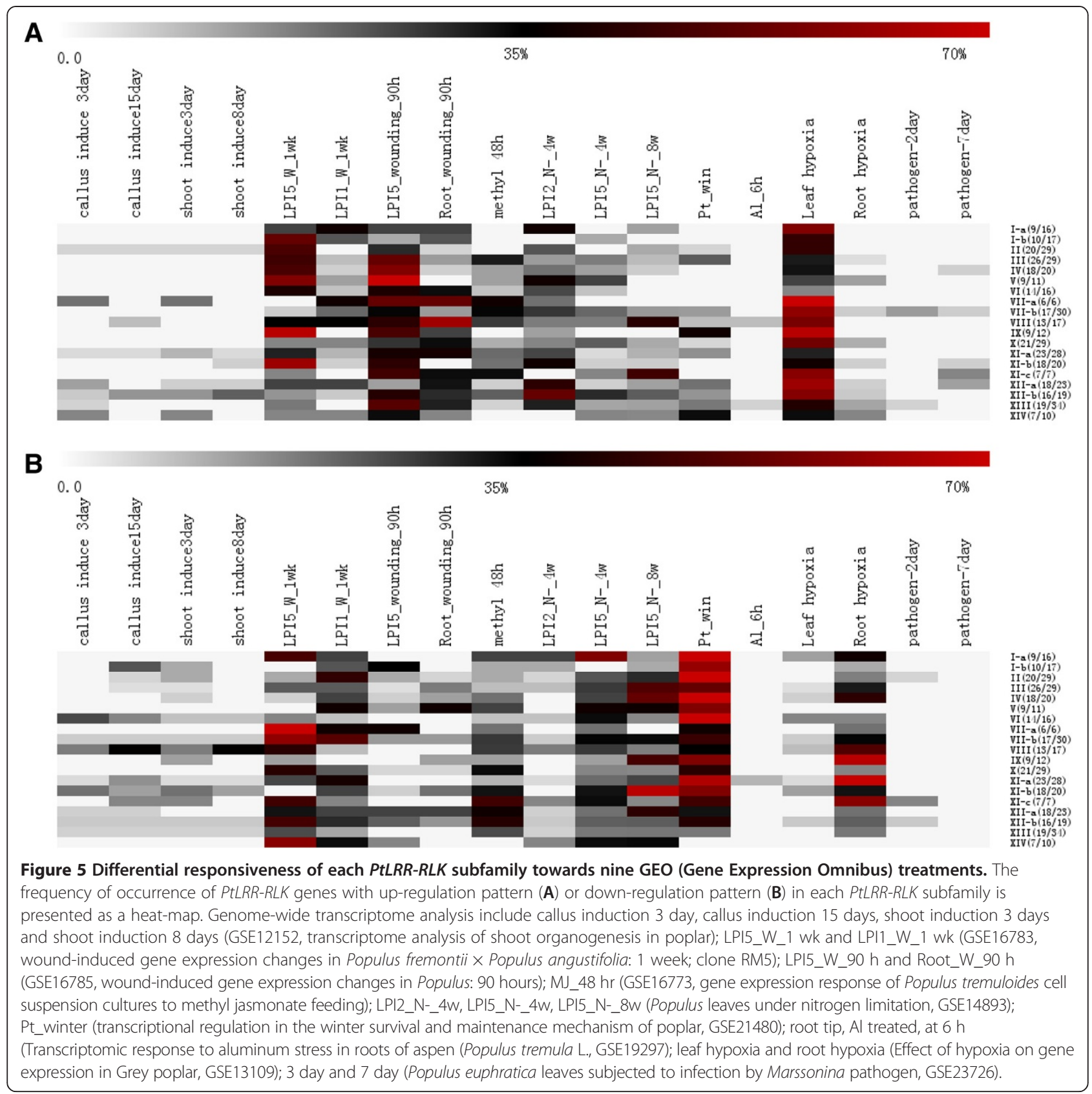

in a tissue-specific manner and responsive to various stresses. Data in this work may provide valuable information for future investigations to reveal the functional divergence and adaptive evolution of this gene family in tree species.

\section{Methods}

Sequence retrieval and phylogenetic reconstruction of LRR-RLK genes in poplar genome

Arabidopsis thaliana gene identifiers of different LRRRLK super-families were downloaded from the PlantsP server v.2011 Arabidopsis 2010 project (http://plantsp. genomics.purdue.edu/html/projects/lrr/Clouse2010.htm) [5] for the first round Blastp search against the poplar genomic sequence database at the DOE Joint Genome Institute (JGI) website. Subsequently, each identified hit was used as a query to conduct Blastp searches in the poplar assembly genomic sequence database to minimize the risk of missing potential PtLRR-RLKs. The version 2.2 P. trichocarpa genome and protein sequences were downloaded from Phytozome (http://www.phytozome. net) [52]. These resulted hit sequences were then 
analyzed with SMART (http://smart.embl-heidelberg.de) [53] and PFAM (http://pfam.sanger.ac.uk/) [54] to assure the presence of at least two LRR domains and one RLK domain. Identical and defective sequences were eliminated using manual inspection in Molecular Evolutionary Genetics Analysis (MEGA) v5.1 [55]. After the signal sequences were deleted, ClustalX v.2.0.12 [56] was used to generate a multiple sequence alignment of either the full length sequences, the trimmed LRR domains or kinase domains among the PtLRR-RLK protein sequences. The phylogenetic trees were constructed using the neighbor-joining method [57] in the MEGA package v5.1 [55] with bootstrap values from 1000 replicates indicated at each node. Representative sequences from each Arabidopsis LRR-RLK subfamily or AtLRR-RLKs with defined functions were chosen to generate alignments with Populus LRR-RLKs.

\section{Protein structure and conserved motif distribution}

The number and position of exons and introns for individual PtLRR-RLK genes were determined by comparison of the cDNAs with their corresponding genomic DNA sequences. Information concerning PtLRR-RLK protein sequences, such as number of amino acids, molecular weights and PIs, were determined using ProtParam (http://au.expasy.org/tools/protparam.html) [58]. Presence of the signal peptides were predicted at SignalP v.4.1 (http://www.cbs.dtu.dk/services/SignalP) [59]. Transmembrane domains were predicted with TMHMM v. 2.0 (http://www.cbs.dtu.dk/services/TMHMM-2.0/) [60] and Phobius (http://phobius.binf.ku.dk/) [61]. To exhibit the structural divergence of PtLRR-RLK genes, the conserved motifs in the encoded proteins were performed with the Multiple Expectation Maximization for Motif Elicitation (MEME) online program v.4.9.0 (http://meme.sdsc.edu/ meme/intro.html) [37] and visualized with WebLogo (http://weblogo.berkeley.edu/logo.cgi) [62]. Parameters were set as follows: the maximum number of motifs 30 ; minimum motif width 10 ; and maximum motif width 30 ; all other parameters were defaulted.

\section{Chromosome location analysis}

The chromosomal locations of the poplar LRR-RLK genes were drawn on the schematic diagram tool at PopGenIE [63] (http://popgenie.org/gp). Identification of homologous chromosome segments resulting from whole-genome duplication events was accomplished as described previously [17]. Blocks with the same color represent homologous chromosome segments. Tandem gene duplications were identified as genes separated by ten or fewer gene loci in a range of $200 \mathrm{~kb}$ distance.

\section{Gene expression analysis}

Gene expression data mainly came from Poplar eFP Browser (http://bar.utoronto.ca/efppop/cgi-bin/efpWeb. cgi). In addition, the gene expression pattern of Populus meristem tissue series was obtained from PopGenIE [63]. The genome-wide microarray data was obtained from the Gene Expression Omnibus database at the NCBI under the series accession numbers GSE23637 (Populus euphratica leaves subjected to drought), GSE13043 (from P. trichocarpa), GSE21480 (transcriptional regulation in the winter survival), GSE20061 (young differentiating xylem of poplar in response to a drought -rewatering cycle), GSE23726 (Populus euphratica leaves subjected to infection by Marssonina pathogen), GSE9673 (interactions with Melampsora rust fungi), GSE13109 (Effect of hypoxia on gene expression in Grey poplar), GSE14893 (Populus leaves under nitrogen limitation: clone 3200), GSE19297 (aluminum stress in roots of aspen, Populus tremula L.), GSE16773 (gene expression response of Populus tremuloides cell suspension cultures to methyl jasmonate feeding), GSE12152 (Genome scale transcriptome analysis of shoot organogenesis in Populus tremula x P. alba), GSE17223 (Molecular bases of acclimation and adaptation to water deficit in Populus anadensis) and GSE16785 (Wound-induced gene expression changes in Populus: 90 hours; clone RM5). Probe sets corresponding to the putative Populus LRR-RLKs were identified using an online Probe Match tool available at the NetAffx Analysis Center (http://www.affymetrix.com/). Genes were clustered based on the expression profiles and Hierarchical clustering of microarray data performed in MultiExperiment Viewer (MeV) v4.7.4 [64], using Pearson correlation and Average Linkage Clustering algorithm. Heatmaps of gene expression were generated using $\mathrm{R}$ (http://www.r-project.org/).

\section{Additional files}

Additional file 1: A complete list of 379 PtLRR-RLKs identified in the present study. Genomic DNA sequences are obtained from Phytozome (http://www.phytozome.net/poplar, release 2.1). Amino acid sequences are deduced from the corresponding coding sequences.

Additional file 2: Maximum likelihood bootstrap tree phylogeny based on the LRR sequences of LRR-RLK genes in Populus trichocarpa. The unrooted tree was constructed using MEGA 4.0. Numbers at nodes indicate the percentage bootstrap scores and only bootstrap values higher than 50\% from 1,000 replicates are shown.

Additional file 3: Maximum likelihood bootstrap tree phylogeny based on the RLK sequences of $L R R-R L K$ genes in Populus trichocarpa. The unrooted tree was constructed using MEGA 4.0. Numbers at nodes indicate the percentage bootstrap scores and only bootstrap values higher than 50\% from 1,000 replicates are shown.

Additional file 4: Maximum likelihood bootstrap tree phylogeny based on the LRR sequences of $L R R$-RLK genes in Populus trichocarpa and Arabidopsis thaliana. The unrooted tree was constructed using MEGA 4.0. Numbers at nodes indicate the percentage bootstrap scores and only bootstrap values higher than $50 \%$ from 1,000 replicates are shown.

Additional file 5: The JGI gene model of each PtLRR-RLK gene to illustrate the distribution and position of introns. Exons and introns are represented to scale by colored boxes and lines, respectively. The 
group number and name of PtLRR-RLK gene and its intron-exon structure pattern are indicated at the left and right sides, respectively.

Additional file 6: Comparison of intron/exon structures between AtLRR-RLKs with known functions and Populus homologues with similar genetic structures.

Additional file 7: Schematic illustrations of the types and distributions of LRR motifs in each Populus LRR-RLK subfamily.

Additional file 8: Non-LRR motifs identified in the extracellular regions of PtLRR-RLKs.

Additional file 9: PtLRR-RLKs sharing the same or similar motif composition and arrangement as AtLRR-RLKs (50 out of 63 with known functions). The motif characterization was based on full-length proteins.

Additional file 10: Locations of tandem clustering on chromosomes and their distribution among fourteen phytogenetic groups.

Additional file 11: Tissue-specific expression patterns of 283 out of 379 PtLRR-RLKs with probes available in the PopGenExpress data set. The microarray-based expression data were downloaded from the Poplar eFP browser, gene-wise normalized and hierarchical clustered based on Pearson correlation. Color scale at the top of each dendrogram represents log2 expression values. Rt, roots; $M L$, mature leaves; $Y L$, young leaves; FC, female catkins; MC, male catkins; DX, differentiating xylems.

Additional file 12: Expression patterns of tandem duplicated gene clusters. The microarray-based expression data were downloaded from the Poplar eFP browser, gene-wise normalized and hierarchical clustered based on Pearson correlation. Color scale at the top of each dendrogram represents log2 expression values. Rt, roots; $M L$, mature leaves; $Y L$, young leaves; FC, female catkins; MC, male catkins; DX, differentiating xylems.

\section{Additional file 13: Expression patterns of 82 pairs of PtLRR-RLK} paralogs. The microarray-based expression data were downloaded from the Poplar eFP browser, gene-wise normalized and hierarchical clustered based on Pearson correlation. Color scale at the top of each dendrogram represents log2 expression values. Rt, roots; $M L$, mature leaves; $Y L$, young leaves; FC, female catkins; MC, male catkins; DX, differentiating xylems.

Additional file 14: Comparison of tissue expression patterns of Arabidopsis LRR-RLKs and their respective Populus homologues.

Additional file 15: Description of Gene Expression Omnibus (GEO) datasets at NCBI website used in this study.

Additional file 16: Populus LRR-RLK genes exhibit differential expression upon a range of treatments. The patterns of relative transcript accumulation of each PtLRR-RLK genes as determined by microarray analysis are presented as a heat map, with red indicating higher levels and blue indicating lower levels of transcript accumulation.

Additional file 17: The percentages of PtLRR-RLK genes from each subfamily being induced (A) or suppressed (B) for each treatment.

Additional file 18: Information on the AGI code, gene full name and abbreviation for each AtLRR-RLK gene with defined functions presented in this work.

\section{Competing interests}

The authors declare that they have no competing interests.

\section{Authors' contributions}

$Y Z$ and $Y J$ performed most of the data mining and data analysis. YZ participated in the illustrations of the figures and tables. SY and YS helped to retrieve data from GEO database and draw the heat-map. JW designed and coordinated the work and wrote the manuscript. All authors read and approved the final manuscript.

\section{Acknowledgements}

This study was supported by National Program on Key Basic Research Project (2012CB114500), National High Technology Research and Development Program (2011AA100200) and National Natural Science Foundation (31270644, 31070600 and 31100451) of China.
Received: 14 February 2013 Accepted: 3 May 2013

Published: 10 May 2013

\section{References}

1. van der Geer P, Hunter T, Lindberg RA: Receptor protein-tyrosine kinases and their signal transduction pathways. Annu Rev Cell Biol 1994, 10:251-337.

2. Walker JC, Zhang R: Relationship of a putative receptor protein kinase from maize to the S-locus glycoproteins of brassica. Nature 1990, 345(6277):743-746.

3. Dievart A, Clark SE: Using mutant alleles to determine the structure and function of leucine-rich repeat receptor-like kinases. Curr Opin Plant Biol 2003, 6(5):507-516

4. Shiu SH, Bleecker AB: Plant receptor-like kinase gene family: diversity, function, and signaling. Sci STKE 2001, 2001(113):re22.

5. Gou X, He K, Yang H, Yuan T, Lin H, Clouse SD, Li J: Genome-wide cloning and sequence analysis of leucine-rich repeat receptor-like protein kinase genes in arabidopsis thaliana. BMC Genomics 2010, 11:19.

6. Afzal AJ, Wood AJ, Lightfoot DA: Plant receptor-like serine threonine kinases: roles in signaling and plant defense. Mol Plant Microbe Interact 2008, 21(5):507-517.

7. Torii KU, Mitsukawa N, Oosumi T, Matsuura Y, Yokoyama R, Whittier RF, Komeda $Y$ : The arabidopsis ERECTA gene encodes a putative receptor protein kinase with extracellular leucine-rich repeats. Plant Cell 1996, 8(4):735-746.

8. Godiard L, Sauviac L, Torii KU, Grenon O, Mangin B, Grimsley NH, Marco Y: ERECTA, an LRR receptor-like kinase protein controlling development pleiotropically affects resistance to bacterial wilt. Plant J 2003, 36(3):353-365.

9. Dievart A, Clark SE: LRR-containing receptors regulating plant development and defense. Development 2004, 131(2):251-261.

10. Mayer KFX, Schoof H, Haecker A, Lenhard M, Jurgens G, Laux T: Role of WUSCHEL in regulating stem cell fate in the arabidopsis shoot meristem. Cell 1998, 95(6):805-815.

11. Schoof H, Lenhard M, Haecker A, Mayer KFX, Jurgens G, Laux T: The stem cell population of arabidopsis shoot meristems is maintained by a regulatory loop between the CLAVATA and WUSCHEL genes. Cell 2000, 100(6):635-644.

12. Brand U, Fletcher JC, Hobe M, Meyerowitz EM, Simon R: Dependence of stem cell fate in arabidopsis on a feedback loop regulated by CLV3 activity. Science 2000, 289(5479):617-619.

13. Deyoung BJ, Clark SE: BAM receptors regulate stem cell specification and organ development through complex interactions with CLAVATA signaling. Genetics 2008, 180(2):895-904

14. Walsh B: Population-genetic models of the fates of duplicate genes. Genetica 2003, 118(2-3):279-294.

15. Shiu SH, Bleecker AB: Expansion of the receptor-like kinase/pelle gene family and receptor-like proteins in arabidopsis. Plant Physiol 2003, 132(2):530-543.

16. Shiu SH, Karlowski WM, Pan R, Tzeng YH, Mayer KF, Li WH: Comparative analysis of the receptor-like kinase family in arabidopsis and rice. Plant Cell 2004, 16(5):1220-1234.

17. Tuskan GA, Difazio S, Jansson S, Bohlmann J, Grigoriev I, Hellsten U, Putnam N, Ralph S, Rombauts S, Salamov A, et al: The genome of black cottonwood, populus trichocarpa (torr. \& Gray). Science 2006, 313(5793): 1596-1604.

18. Dievart A, Gilbert N, Droc G, Attard A, Gourgues M, Guiderdoni E, Perin C: Leucine-rich repeat receptor kinases are sporadically distributed in eukaryotic genomes. BMC Evol Biol 2012, 11:367.

19. Sun X, Wang GL: Genome-wide identification, characterization and phylogenetic analysis of the rice LRR-kinases. PLoS One 2011, 6(3):e16079

20. Lehti-Shiu MD, Zou C, Hanada K, Shiu SH: Evolutionary history and stress regulation of plant receptor-like kinase/pelle genes. Plant Physiol 2009, 150(1):12-26.

21. Shiu SH, Li WH: Origins, lineage-specific expansions, and multiple losses of tyrosine kinases in eukaryotes. Mol Biol Evol 2004, 21(5):828-840.

22. Tanaka M, Takahata Y, Nakayama H, Nakatani M, Tahara M: Altered carbohydrate metabolism in the storage roots of sweet potato plants overexpressing the SRF1 gene, which encodes a Dof zinc finger transcription factor. Planta 2009, 230(4):737-746.

23. Eyuboglu B, Pfister K, Haberer G, Chevalier D, Fuchs A, Mayer KF, Schneitz K: Molecular characterisation of the STRUBBELIG-RECEPTOR FAMILY of genes encoding putative leucine-rich repeat receptor-like kinases in arabidopsis thaliana. BMC Plant Biol 2007, 7:16. 
24. Dolan L: Positional information and mobile transcriptional regulators determine cell pattern in the arabidopsis root epidermis. J Exp Bot 2006, 57(1):51-54

25. Kinoshita T, Cano-Delgado A, Seto H, Hiranuma S, Fujioka S, Yoshida S, Chory J: Binding of brassinosteroids to the extracellular domain of plant receptor kinase BRI1. Nature 2005, 433(7022):167-171.

26. van Zanten M, Snoek LB, Proveniers MC, Peeters AJ: The many functions of ERECTA. Trends Plant Sci 2009, 14(4):214-218.

27. Sanchez-Rodriguez C, Estevez JM, Llorente F, Hernandez-Blanco C, Jorda L, Pagan I, Berrocal M, Marco Y, Somerville S, Molina A: The ERECTA receptorlike kinase regulates cell wall-mediated resistance to pathogens in arabidopsis thaliana. Mol Plant Microbe Interact 2009, 22(8):953-963.

28. Wang Z, Meng P, Zhang X, Ren D, Yang S: BON1 Interacts with the protein kinases BIR1 and BAK1 in modulation of temperature-dependent plant growth and cell death in arabidopsis. Plant J 2011, 67(6):1081-1093.

29. Lee JS, Kuroha T, Hnilova M, Khatayevich D, Kanaoka MM, McAbee JM, Sarikaya M, Tamerler C, Torii KU: Direct interaction of ligand-receptor pairs specifying stomatal patterning. Genes Dev 2012, 26(2):126-136

30. Yamaguchi $Y$, Huffaker A, Bryan AC, Tax FE, Ryan CA: PEPR2 Is a second receptor for the Pep1 and Pep2 peptides and contributes to defense responses in arabidopsis. Plant Cell 2010, 22(2):508-522.

31. Krol E, Mentzel T, Chinchilla D, Boller T, Felix G, Kemmerling B, Postel S, Arents M, Jeworutzki E, Al-Rasheid KA, et al: Perception of the arabidopsis danger signal peptide 1 involves the pattern recognition receptor AtPEPR1 and its close homologue AtPEPR2. J Biol Chem 2010, 285(18): 13471-13479.

32. Gomez-Gomez L, Boller T: FLS2: an LRR receptor-like kinase involved in the perception of the bacterial elicitor flagellin in arabidopsis. $\mathrm{Mol} \mathrm{Cell}$ 2000, 5(6):1003-1011.

33. Zipfel C, Kunze G, Chinchilla D, Caniard A, Jones JD, Boller T, Felix G. Perception of the bacterial PAMP EF-Tu by the receptor EFR restricts agrobacterium-mediated transformation. Cell 2006, 125(4):749-760.

34. Zhao DZ, Wang GF, Speal B, Ma H: The excess microsporocytes1 gene encodes a putative leucine-rich repeat receptor protein kinase that controls somatic and reproductive cell fates in the arabidopsis anther. Genes Dev 2002, 16(15):2021-2031.

35. Wang J, Tan S, Zhang L, Li P, Tian D: Co-variation among major classes of LRR-encoding genes in two pairs of plant species. J Mol Evol 2011, 72(56):498-509.

36. Karve R, Liu W, Willet SG, Torii KU, Shpak ED: The presence of multiple introns is essential for ERECTA expression in arabidopsis. RNA 2011, 17(10):1907-1921.

37. Bailey TL, Williams N, Misleh C, Li WW: MEME: discovering and analyzing DNA and protein sequence motifs. Nucleic Acids Res 2006, 34(Web Server issue):W369-W373.

38. Padmanabhan $M$, Cournoyer $P$, Dinesh-Kumar SP: The leucine-rich repeat domain in plant innate immunity: a wealth of possibilities. Cell Microbiol 2009, 11(2):191-198.

39. Kobe B, Deisenhofer J: The leucine-rich repeat: a versatile binding motif. Trends Biochem Sci 1994, 19(10):415-421.

40. Ellis J, Dodds P, Pryor T: The generation of plant disease resistance gene specificities. Trends Plant Sci 2000, 5(9):373-379.

41. Hanks SK, Hunter T: Protein kinases 6. The eukaryotic protein kinase superfamily: kinase (catalytic) domain structure and classification. FASEB $J$ 1995, 9(8):576-596.

42. Adams JA: Activation loop phosphorylation and catalysis in protein kinases: is there functional evidence for the autoinhibitor model? Biochemistry 2003, 42(3):601-607.

43. Krupa A, Preethi G, Srinivasan N: Structural modes of stabilization of permissive phosphorylation sites in protein kinases: distinct strategies in Ser/Thr and Tyr kinases. J Mol Biol 2004, 339(5):1025-1039.

44. Dardick C, Ronald P: Plant and animal pathogen recognition receptors signal through non-RD kinases. PLoS Pathog 2006, 2(1):e2.

45. Hwang SG, Kim DS, Jang CS: Comparative analysis of evolutionary dynamics of genes encoding leucine-rich repeat receptor-like kinase between rice and arabidopsis. Genetica 2011, 139(8):1023-1032.

46. Holub EB: The arms race is ancient history in arabidopsis, the wildflower. Nat Rev Genet 2001, 2(7):516-527.

47. Wilkins O, Nahal H, Foong J, Provart NJ, Campbell MM: Expansion and diversification of the populus R2R3-MYB family of transcription factors. Plant Physiol 2009, 149(2):981-993.
48. Yim WC, Lee BM, Jang CS: Expression diversity and evolutionary dynamics of rice duplicate genes. Mol Genet Genomics 2009, 281(5):483-493.

49. Blanc $\mathrm{G}$, Wolfe $\mathrm{KH}$ : Functional divergence of duplicated genes formed by polyploidy during arabidopsis evolution. Plant Cell 2004, 16(7):1679-1691.

50. Chen F, Mackey AJ, Stoeckert CJ Jr, Roos DS: OrthoMCL-DB: querying a comprehensive multi-species collection of ortholog groups. Nucleic Acids Res 2006, 34(Database issue):D363-D368.

51. Sonnhammer EL, Koonin EV: Orthology, paralogy and proposed classification for paralog subtypes. Trends Genet 2002, 18(12):619-620.

52. Goodstein DM, Shu S, Howson R, Neupane R, Hayes RD, Fazo J, Mitros T, Dirks W, Hellsten U, Putnam N, et al: Phytozome: a comparative platform for green plant genomics. Nucleic Acids Res 2012, 40(Database issue): D1178-D1186.

53. Schultz J, Copley RR, Doerks T, Ponting CP, Bork P: SMART: a web-based tool for the study of genetically mobile domains. Nucleic Acids Res 2000, 28(1):231-234.

54. Finn RD, Tate J, Mistry J, Coggill PC, Sammut SJ, Hotz HR, Ceric G, Forslund K, Eddy SR, Sonnhammer EL, et al: The pfam protein families database. Nucleic Acids Res 2008, 36(Database issue):D281-D288.

55. Tamura K, Peterson D, Peterson N, Stecher G, Nei M, Kumar S: MEGA5: molecular evolutionary genetics analysis using maximum likelihood, evolutionary distance, and maximum parsimony methods. Mol Biol Evol 2011, 28(10):2731-2739.

56. Chenna R, Sugawara H, Koike T, Lopez R, Gibson TJ, Higgins DG, Thompson JD: Multiple sequence alignment with the clustal series of programs. Nucleic Acids Res 2003, 31(13):3497-3500.

57. Saitou N, Nei M: The neighbor-joining method: a new method for reconstructing phylogenetic trees. Mol Biol Evol 1987, 4(4):406-425.

58. Wilkins MR, Gasteiger E, Bairoch A, Sanchez JC, Williams KL, Appel RD, Hochstrasser DF: Protein identification and analysis tools in the ExPASy server. Methods Mol Biol 1999, 112:531-552.

59. Petersen TN, Brunak S, von Heijne G, Nielsen H: SignalP 4.0: discriminating signal peptides from transmembrane regions. Nat Methods 2011, 8(10):785-786.

60. Krogh A, Larsson B, von Heijne G, Sonnhammer EL: Predicting transmembrane protein topology with a hidden markov model: application to complete genomes. J Mol Biol 2001, 305(3):567-580.

61. Kall L, Krogh A, Sonnhammer EL: Advantages of combined transmembrane topology and signal peptide prediction--the phobius web server. Nucleic Acids Res 2007, 35(Web Server issue):W429-W432.

62. Crooks GE, Hon G, Chandonia JM, Brenner SE: WebLogo: a sequence logo generator. Genome Res 2004, 14(6):1188-1190.

63. Sjodin A, Street NR, Sandberg G, Gustafsson P, Jansson S: The populus genome integrative explorer (PopGenIE): a new resource for exploring the populus genome. New Phyto/ 2009, 182(4):1013-1025.

64. Saeed Al, Sharov V, White J, Li J, Liang W, Bhagabati N, Braisted J, Klapa M, Currier T, Thiagarajan M, et al: TM4: a free, open-source system for microarray data management and analysis. Biotechniques 2003, 34(2):374-378.

doi:10.1186/1471-2164-14-318

Cite this article as: Zan et al: Genome-wide identification,

characterization and expression analysis of populus leucine-rich repeat receptor-like protein kinase genes. BMC Genomics 2013 14:318.

\section{Submit your next manuscript to BioMed Central and take full advantage of:}

- Convenient online submission

- Thorough peer review

- No space constraints or color figure charges

- Immediate publication on acceptance

- Inclusion in PubMed, CAS, Scopus and Google Scholar

- Research which is freely available for redistribution 